\section{A SEGURANÇA BIOMECÂNICA NA PRÁTICA CLINICA DOS ENFERMEIROS ESPECIALISTAS EM SAÚDE MATERNA E OBSTETRÍCIA}

\author{
Biomechanical safety in the clinical practice of nurses \\ specializing in maternal health and obstetrics
}

\section{RESUMO}

Objetivo: Compreender as medidas preventivas das lesões músculo-esqueléticas ligadas ao trabalho integradas pelos enfermeiros especialistas de saúde materna e obstétrica, na sua prática clinica bem como as dificuldades em adotá-las ou mantê-las. Métodos: Neste estudo exploratório e descritivo de abordagem qualitativa, privilegiou-se o questionário composto por questões abertas que foram sujeitas à técnica de analise de conteúdo. Selecionou-se uma amostra por conveniência: enfermeiros com experiência profissional em salas de parto de hospitais de cuidados diferenciados perinatais. Resultados: Participaram 14 enfermeiros especialistas de saúde materna e obstétrica, maioritariamente do sexo feminino, média de idades 37,2 anos e 21,3 anos de atividade profissional. Os participantes do estudo valorizam como medidas preventivas de LMELT o controlo do ambiente físico (organização do espaço e manipulação de materiais e equipamentos), a preparação da parturiente (avaliação da capacidade de colaboração, explicitar o procedimento, antecipar movimentos e prever o comportamento da mulher), as práticas e comportamentos no período expulsivo (recurso aos princípios da biomecânica, postura estática versus postura dinâmica, segurança da parturiente e bem-estar do recém-nascido) e as práticas e comportamentos no período pósparto (atividade/repouso; stress pós-parto e comportamento individual). Conclusão: A prevalência de lesões músculo-esqueléticas nos enfermeiros especialistas de saúde materna e obstétrica é elevada, com impacto económico no tratamento e diminuição da produtividade. Verifica-se uma maior preocupação com o conforto da parturiente/RN em detrimento da segurança profissional. A postura varia com o tipo de parto, as dificuldades em manter os princípios da biomecânica são influenciadas pelos espaço físico e equipamentos.

Descritores: Enfermeiros Obstetras; Prevenção e Controle; Transtornos Traumáticos Cumulativos.

\section{ABSTRACT}

Objective: Understand the preventive measures of the work-related musculoskeletal disorders integrated by nurses specialists in maternal health and obstetrics, in the clinic well practice as well as the difficulties to adopt or keep them. Methods: In this exploratory study and descriptive quality approach, focused on the questionnaire composed by open questions that were subjected to the content analysis technique. It was selected a sample by convenience: the nurses with professional experience that worked on the delivery rooms of hospitals with perinatal differentiated care. Results: Fourteen nurse specialists in maternal health and obstetrics, majorly from the feminine gender, with an age average of 37.2 and 21.3 years of professional experience participated on this questionnaire. Study participants value as preventive measures of the WMSDs physical environment control (organization of space and materials handling and equipment), parturient preparation (assessment of collaboration capabilities, clarify the procedure to anticipate movements and predict the behavior of women), practices and behaviours during the second stage (using the principles of biomechanics, static posture versus dynamic posture, safety of the mother and well-being of the newborn) and practices and behaviours during the after birth period (activity/rest; postpartum stress and individual behavior). Conclusion: The work-related musculoskeletal disorders (WMSDs) prevalence in nurse specialists in maternal health and obstetrics is high, with an economic impact in the treatment and decreased productivity. It is observed a higher concern about the parturient/newborn comfort in detriment of professional safety. The posture varies with the type of labor, the difficulties to maintain the biomechanical principles are influenced by the physical spaces and equipments.

Descriptors: Nurse Midwives; Prevention e Control; Cumulative Trauma Disorders.
Artigo Original
Cristina Lavareda Baixinho(1)

Helena Presado ${ }^{(1)}$

Fátima Mendes Marques ${ }^{(1)}$ Mário Cardoso(1)

1) Escola Superior de Enfermagem de Lisboa - ESEL- Lisboa - Portugal

Este artigo seguiu as normas e formatação estabelecidas pelo $5^{\circ}$ CIAIQ - Congresso Ibero-Americano em Investigação Qualitativa. 


\section{INTRODUÇÃO}

As lesões músculo esqueléticas ligadas ao trabalho (LMELT) representam um grave problema de saúde ocupacional para os profissionais de saúde que exercem a sua atividade profissional em hospitais ${ }^{(1)}$.

A prevalência varia de $35 \%$ a $84,31 \%^{(1-4)}$, com uma elevada percentagem da população $\left(65,4 \%^{(1)}-71,9 \%^{(5)}\right)$ a experienciar uma LMLET nos últimos 12 meses $^{(1)}$. A dor e o desconforto músculo-esquelético são uma queixa presente desde os primeiros anos de atividade profissional ${ }^{(6)}$, causam diversas complicações físicas e psicológicas, provocando absentismo $^{(5)}$ e representam $56 \%$ das causas de ausência temporária ao serviço ${ }^{(1)}$.

A discrepância nos valores da prevalência encontrada, nos diferentes estudos, pode ter sido influenciada pelos métodos, técnicas, procedimentos e tamanho da amostra, mas revelam a magnitude de um problema de saúde ocupacional que demanda um resolução urgente.

Epidemiológica e ergonomicamente as LMELT conquistaram uma posição de destaque nas políticas de saúde ocupacional pelo elevado impacto económico no tratamento das lesões e diminuição da produtividade ${ }^{(1)}$. O aumento da esperança média de vida, com a consequente tradução no aumento do tempo útil de vida produtiva, exige medidas que garantam qualidade e segurança nos locais de trabalho, não só para os clientes, mas também para os profissionais. Porque se por um lado a população necessita de cuidados seguros e de qualidade, por outro, os trabalhadores da enfermagem também precisam de condições organizacionais favoráveis para desenvolver o seu papel que é de suma importância para todos os que vivem em sociedade ${ }^{(7)}$.

Este é um fenómeno complexo pela multifatorialidade na sua génese. As causas incluem riscos individuais, comportamentais, organizacionais e arquitectónicos. Destacam-se o desrespeito pelos princípios da biomecânica com posturas inadequadas mantidas por longos períodos, a não garantia de um alinhamento corporal correto durante a execução das atividades, a não utilização das alavancas corporais para a mobilização de cargas $^{(1)}$; o uso excessivo da força ${ }^{(1,3-4)}$; os ritmos de trabalhos intensos; o estresse; as atividades de posicionamento, levante e transferência de pessoas dependentes ${ }^{(4-5,8)}$; mobilização de $\operatorname{cargas}^{(1,7)}$; a falta de informação e treino dos profissionais para a execução das atividades em segurança ${ }^{(6)}$; problemas de saúde, falta de exercício físico e a obesidade ${ }^{(1)}$.

A maioria dos estudos avalia, este fenómeno, descriminando as categorias profissionais (enfermeiros, médicos, auxiliares, fisioterapeutas, entre outros) e o tipo de lesões (lesões de esforço repetitivo, tendinites, dorsolombalgias, dor/desconforto $)^{(1-6,8)}$.

A revisão da literatura permite afirmar que existem poucos estudos sobre a prevalência de diferentes tipos de LMELT entre os enfermeiros especialistas de saúde materna e obstétrica (EESMO) comparativamente com médicos e enfermeiros que exercem atividade em urgências, cuidados intensivos e serviços de internamento de pessoas adultas e $\operatorname{idosas}^{(9)}$.

A investigação tem sido produtiva sobre os fatores de risco, todavia a etiologia de algumas lesões e acidentes profissionais não está completamente clara $^{(1)}$. Os investigadores consideram que os estudos que envolvem o risco ocupacional nos hospitais centram-se muito nas atividades relacionadas com a prestação direta de cuidados aos doentes ${ }^{(4)}$, não explorando outras variáveis importantes, que permitam uma compreensão mais abrangente deste problema $^{(8)}$ de saúde pública, o que influencia a prevenção, sobretudo para as causas relacionadas com o comportamento humano. Há poucos artigos que analisam a eficácia das diferentes estratégias/programas implementados com o objetivo de prevenir ou reduzir as LMELT ${ }^{(1)}$ nos EESMO.

Face ao estado da arte, a finalidade deste estudo é compreender de que forma o enfermeiro especialista de saúde materna e obstétrica integra, na sua prática clínica, a "segurança biomecânica" para prevenir as LMELT.

\section{MÉTODOS}

A abordagem desta investigação é qualitativa, de carácter exploratório e descritivo. A importância do trabalho qualitativo no desenvolvimento do conhecimento em enfermagem defende-se pelo questionamento e descrição dos fenómenos, permitindo uma interpretação que leva à compreensão dos dados e consequente teoria ${ }^{(10)}$. A opção metodológica prende-se com o estado da arte em relação às medidas preventivas utilizadas pelos EESMO para evitarem as LMELT, e porque o primeiro passo para a implementação de programas de prevenção, para além da identificação do risco, é compreender quais as práticas preventivas Neste sentido, compreender as medidas preventivas das LMELT integradas pelos enfermeiros especialistas de saúde materna e obstétrica, na sua prática clinica bem como as dificuldades em adotá-las ou mantê-las, é o objetivo do nosso estudo.

A avaliação, tanto do risco como das medidas preventivas, de LMELT incide nas diferentes expectativas dos EESMO, considerando pontos de vista e práticas distintas, sobre a forma como conhecem e compreendem a problemática de $\operatorname{LMELT}^{(10)}$.

Partindo do pressuposto que a segurança biomecânica na prática clínica dos enfermeiros especialistas em saúde 
materna e obstetrícia constitui um mundo complexo, considerou-se necessário para a sua interpretação, questionar os EESMO que desenvolvessem a sua atividade profissional em sala de partos, sobre os factores de risco e as medidas preventivas que adoptavam no seu quotidiano.

Para a colheita dos achados elaborou-se um questionário composto por duas partes, a primeira permitia a caracterização sociodemográfica e a segunda por questões abertas, de modo a permitir que os participantes pensarem sobre o desejavam partilhar ${ }^{(10)}$, desenvolvido com base na literatura, com as devidas adaptações à especificidade da atividade profissional dos participantes.

A amostra de conveniência foi constituída por 14 EESMO, que respeitavam os critérios de inclusão definidos: terem mais de cinco anos de atividade profissional e exercerem a sua atividade profissional em sala de partos.

Os profissionais foram contactados individualmente por um dos investigadores durante o terceiro trimestre de 2015, solicitando-se a participação no preenchimento do questionário e garantindo o anonimato e a confidencialidade dos dados. Foi dada informação sobre o grupo de investigadores, a finalidade e as formas de divulgação.
No tratamento dos dados, a análise e atribuição de significado, bem como a interpretação, foram realizadas através de uma leitura não 'à letra', mas pelo contrário, em que se procurou a realçar o sentido secundário encoberto ${ }^{(11)}$.

A categorização surgiu, posteriormente, com o compromisso de conseguir estruturar o processo analítico de modo a tornar compreensível o que os dados revelavam ${ }^{(10)}$.

Recorreu-se a dois juízes ao longo do processo de análise para aferir a validade, adequação, pertinência, exaustividade, homogeneidade e objectividade das categorias.

\section{RESULTADOS}

Participaram neste estudo 14 EESMO, maioritariamente mulheres (13), com uma média de idades de 37,2 anos, trabalham na área de especialidade, em média, há 9,6 anos.

A análise do seu discurso permitiu a definição de cinco categorias em relação às medidas preventivas adotadas pelo EESMO. A primeira leitura dos achados, sem preocupação em categorizar, possibilitou inferir que as medidas adotadas rodam em torno do trabalho de parto. Por este motivo optou-se por uma organização categorial cronológica, pela sequência da organização do trabalho de parto (Tabela I),

Tabela I - Corpus da análise de conteúdo. Lisboa; Portugal. 2016.

\begin{tabular}{llc}
\hline Categoria & Subcategoria & $\begin{array}{c}\text { FI (Unidades de } \\
\text { Enumeração) }\end{array}$ \\
\hline \multirow{3}{*}{ Controlo do Ambiente Físico da Sala de Partos } & Organização do espaço físico & 17 \\
& Manipulação de materiais e equipamentos & 11 \\
& Subtotal & $\mathbf{2 8}$ \\
& Avalia a capacidade de colaboração da mulher & 15 \\
& Explicita o procedimento & 14 \\
& Antecipa movimentos & 8 \\
& Prevê o comportamento da mulher & 5 \\
& Subtotal & $\mathbf{4 2}$ \\
& Princípios da biomecânica & 11 \\
& Postura estática versus dinâmica & 4 \\
Práticas e Comportamentos no Período Expulsivo Parturiente & Segurança da parturiente & 8 \\
& Bem-estar do recém-nascido & 6 \\
& Subtotal & $\mathbf{2 9}$ \\
& Atividade/Repouso & 3 \\
& Stress pós-parto & 2 \\
Práticas e Comportamentos Período Pós-Parto & Comportamento individual & 3 \\
& Subtotal & $\mathbf{8}$ \\
& Total & 107 \\
\hline
\end{tabular}


identificando-se quatro períodos chave para práticas de prevenção: 1) controlo do ambiente físico da sala de partos; 2) preparação da parturiente; 3) período expulsivo e 4) período pós-parto.

A consciência de que a prevalência de LMELT é elevada justifica a organização do espaço físico e a manipulação de materiais e equipamentos ao longo do turno de trabalho. $\mathrm{Na}$ subcategoria organização do espaço físico emerge que esta é uma atividade secundaria dependente de outras variáveis como a segurança da parturiente "quando estou na sala de partos a primeira preocupação se não tiver uma mulher em trabalho de parto é organizar a sala(...)" (E5), somente "quando não há partos de seguida há possibilidade de organizar o espaço da sala retirando equipamentos que não precisamos e reorganizando o espaço físico, porque ao longo do parto as mesas de apoio são mobilizadas de um local para o outro" (E13).

Segundo o ponto de vista das entrevistadas a rotatividade, o desenrolar de etapas criticas no parto e as complicações que não se conseguem antecipar dificultam que esta prática de prevenção das LMELT, seja mantida "esqueço-me de ajustar a altura da superfície de trabalho, a colega do turno anterior saiu da sala e eu substituo-a, se a mulher está a entrar no período expulsivo, a atenção à cliente é tanta que nem me lembro que a mesa também é regulável em altura" (E2).

Os participantes assumem que as dificuldades na organização do espaço e dos equipamentos, ultrapassam a decisão individual ou da equipa, defendendo que a ausência de medidas institucionais e organizacionais tem interferência com a segurança biomecânica dos profissionais. Uma especialista salienta que "há muitas instruções de trabalho para a segurança dos clientes, mas não para os profissionais" (E11), outra colega reforça que "o matéria danificado não é reparado de imediato, por vezes, demora meses até se substituir uma peça avariada do hidráulico da marquesa" (E6), porque "infelizmente não há manutenção adequada dos equipamentos" (E14).

A categoria 'Preparação da Parturiente' é a que tem uma maior expressividade, em termos, de unidades de enumeração, surgindo a preocupação em manter práticas seguras na avaliação da capacidade de colaboração da mulher, na explicação do(s) procedimento(s), na antecipação de movimentos e na previsão do comportamento da mulher.

A análise do discurso deixa transparecer que a prevenção das LMELT pode começar antes do trabalho de parto pelo nível de preparação de cada parturiente. É unânime entre os participantes que a frequência de programas de preparação para o parto influencia a capacidade de colaborar ativamente no trabalho de parto e consideram que na ausência de programas formais de preparação para o parto um investimento na comunicação, com informação, avaliação da capacidade de colaboração da mulher e definição de um conjunto de tarefas que podem ser efetuadas pela parturiente, são cruciais para garantir a confiança no profissional, condicionando o desenrolar do trabalho de parto e com impacto nas condições de trabalho dos profissionais, como ilustra a seguinte afirmação, "ao longo de todas as fases vou dando indicações de como se deve posicionar ou exercer a força, é vital a colaboração da mulher não só para o sucesso do parto, mas também para o esforço físico que temos de fazer" (E14).

O conhecimento intuitivo, alicerçado na experiência, permite que os EESMO possam antever o comportamento da cliente e com isso podem atempadamente adequar as estratégias preventivas de LMELT, "já fiz formação sobre as posturas a adotar, foi importante, mas as estratégias que uso advém muito das experiências que tive. Ao avaliar a dor e a ansiedade da mulher consigo antever possíveis alterações no comportamento que não garantam a colaboração e que implicam maior intervenção do profissional, com o uso de força" (E7).

O período expulsivo, surge no discurso dos enfermeiros, como o momento chave para a adoção dos princípios da biomecânica "dependendo da posição em que a mulher está, tento sempre que possível adoptar uma postura que mantenha o equilíbrio e com o mínimo de esforço muscular ao nível da coluna lombar e cervical" (E5) e para o controlo da postura (estática versus dinâmica), "frequentemente e de uma forma muito rápida, quase instintiva, temos de passar de uma posição para outra, por exemplo, estamos numa posição para avaliar o colo e rapidamente temos de mudar de posição e aplicar força para facilitar o trabalho de parto" (E14).

A adoção dos princípios da biomecânica fica condicionada pela diversidade de atividades executadas "é tudo muito rápido e com vários focos de atenção, o que menos me preocupa é como estou" (E3) e pelo tipo de parto "o que não permite pensar em como nos posicionamos" (E5).

A subcategoria 'segurança da parturiente' surge como uma preocupação transversal às diferentes etapas, com influência na tomada de decisão sobre a segurança biomecânica do profissional porque "não permite pensar em como nos posicionamos, a preocupação é a mulher" (E14).

A aplicação de força necessária para auxiliar na expulsão do bebé, aliada a uma base se sustentação pouco ampla, ao desalinhamento corporal com dorsiflexão e torsão da coluna dorso lombar e aos movimentos descoordenados levam a que após o trabalho de parto o profissional sinta fadiga e desconforto músculo esquelética "posso fazer cinco partos num turno, mas se dois são seguidos, o desconforto lombar e a sensação de fadiga muscular quadruplica" (E5). 
A fadiga justifica que na categoria 'Práticas e Comportamentos Período Pós-Parto' surja a preocupação com a 'atividade/repouso', o 'stress pós-parto' e o 'comportamento individual' como variáveis que condicionam as práticas seguras. Os EESMO afirmam que após o esforço despendido no período expulsivo sentem a necessidade de repousar ou de "andar pelo serviço não só para relaxamento muscular, mas também para desanuviar do stress e da preocupação inerente a todo o processo" (E3).

\section{DISCUSSÃO}

A atividade profissional em sala de partos é complexa com diferentes focos de atenção em simultâneo o que implica um ajuste biomecânico constante tanto nas posição estática, como na dinâmica ${ }^{(12)}$.

A análise das respostas permite aferir que profissionais diferentes valorizam medidas diferentes e apontam dificuldades diferentes à sua adoção ou manutenção, apesar de todos trabalharem em sala de partos.

Os EESMO, ao longo do turno de trabalho, assumem posturas inadequadas, com recurso ao uso da força muscular excessiva, devido não só às demandas profissionais, mas também ao ambiente de trabalho inadequado ${ }^{(13)}$, que se traduz em lesão para cada $3 \mathrm{em} 4$ enfermeiras por ano ${ }^{(5)} \mathrm{e} o$ risco aumenta a partir dos 7 anos de atividade profissional ${ }^{(4)}$.

As posturas das especialistas são influenciadas face ao tipo de parto (em particular os nascimentos de água) ${ }^{(13)}$, pelo tipo de marquesas, camas e outros equipamentos pesados que tem de mobilizar ${ }^{(1,12)}$.

No discurso das profissionais percebe-se que o parto no banco tem vantagens para a mãe, mas exige que a enfermeira tenha que se "agachar" para trabalhar(13), numa postura e alinhamento corporal deficitário, períodos prolongados na posição sentada e de pé, flexão do pescoço acentuada e prolongada, dorsiflexão em carga, movimentos repetitivos e/ou fortes, elevação do membro superior superior a $90^{\circ(14)}$.

É consensual entre os participantes que a existência e frequência de programas de preparação para o parto influencia a capacidade de colaboração da parturiente permitindo ao EESMO antecipar e controlar o processo de uma modo mais efetivo e seguro.

A permanência por longos períodos de pé e as posturas desconfortáveis, aumentando o desvio da coluna por períodos prolongados aumenta a musculatura para vertebral, produzindo lesão muscular ${ }^{(9)}$. O estresse físico e mental $^{(1)}$, relacionado com o trabalho, que é emocionalmente exigente $^{(13)}$; os movimentos repetitivos, muitas vezes em carga para suportar o recém-nascido e a manipulação manual de cargas pesadas ${ }^{(4,12)}$, bem como os recursos humanos e materiais, por vezes, escassos e não adaptados às características antropométricas dos profissionais e a falta de organização no local de trabalho ${ }^{(15)}$, justificam, no discurso das participantes as dificuldades que têm na adoção dos princípios da biomecânica, sobretudo no período expulsivo e em função da posição e da colaboração da parturiente.

Os profissionais referem inclusive que não há uma preocupação na organização do espaço e dos equipamentos, porque a prioridade é a segurança da parturiente e não do profissional $^{(12)}$.

Os achados de outros estudos afirmam que estes profissionais têm riscos específicos associados à natureza da tarefa em si, que implicam que durante alguns procedimentos façam movimentos bruscos de pronosupinação, trabalhem com as mãos elevadas em relação aos ombros, com os ombros em tensão e elevados, e é nesta posição, já penosa em si, que puxam o recém-nacido ${ }^{(5)}$.

Outras atividades requerem que os profissionais assumam posições com desalinhamento postural, desvio lateral da coluna dorsolombar, ou mesmo movimentos que requerem dorsiflexão e/ou torção das vértebras cervicais e lombares por longo períodos de tempo o que aumenta o risco de lesão ${ }^{(3)}$, até porque a realização destes movimentos implicam contração isométrica dos grupos musculares responsáveis pela manutenção da posição corporal estática ${ }^{(3)}$.

Nos movimentos que implicam uma mudança rápida da posição estática para a dinâmica também são efetuadas contrações musculares excêntricas ${ }^{(3)}$, que agravam o risco de lesão, uma especialista relata que a mudança de uma posição estática para uma dinâmica é rápida, quase instintiva o que dificulta planear o movimento e a postura corporal.

De salientar que este é o grupo profissional da saúde com um maior número de lesões no ombro, que são de difícil reabilitação, mesmo quando sujeita a cirurgia, tornandose em incapacidades crónicas e estão entre as lesões com maior impacto nos gastos com a saúde ocupacional ${ }^{(16)}$.

As medidas preventivas valorizadas pelos profissionais, deste estudo, são o planeamento da atividade a realizar, a antecipação de possíveis intercorrências para diminuir o risco de adotar posições incorretas e no pós-parto a existência de períodos de repouso.

No que concerne ao planeamento uma pesquisa refere que esta é uma boa estratégia e ilustra que o trabalhar na frente da mãe e usar um nível de trabalho adequado, facilita a adoção de posturas mais ergonómicas ${ }^{(13)}$. Uma boa posição para além de ser biomecanicamente mais segura, torna possível ver a expressão facial da mãe, avaliar a respiração da parturiente e outros sinais importantes, promove a confiança e pode influenciar positivamente a experiência de dor de uma mulher durante o parto ${ }^{(13)}$. 
Uma postura correta inclui a manutenção dos ombros abaixo de $90^{\circ}$, os membros superiores em adução; os antebraços suportados, com o cotovelo com um ângulo inferior a $90-120^{\circ}$ de flexão e o desvio radial e flexão do punho deve ser mínima, sobretudo em carga ${ }^{(14)}$.

Os programas de educação sobre as medidas preventivas e estratégias de coping a utilizar são recomendados para os profissionais de saúde hospitalares para reduzir a prevalência de lesões ${ }^{(1,6)}$ e, simultaneamente para promover cuidados eficientes aos clientes ${ }^{(1)}$. A formação sobre os princípios da biomecânica e a sua aplicação durante as atividades mais penosas como transferência e manipulação das parturientes são medidas preventivas ${ }^{(3)}$. A evidência demonstra que quando a técnica de mobilização da parturiente é aplicada, o risco de LMELT diminui (sobretudo nas lesões ao nível da região dorsolombar), o que reforça a implementação de estratégias eficazes que promovam a adopção dos princípios da biomecânica ${ }^{(17)}$ no que respeita ao movimento, à aplicação da força, à postura e ao alinhamento dos segmentos corporais.

Contudo na resposta às questões, os EESMO referem as medidas preventivas, mas colocam sempre uma referência, ou mais, às dificuldades que sentem na assunção de práticas e comportamentos seguros. As dificuldades não aparecem associadas à falta de formação, mas sim em relação às condicionantes físicas, organizacionais e associadas à natureza da tarefa.

Os programas de prevenção das LMELT, nos hospitais, devem fazer parte da política organizacional e das medidas de melhoria do ambiente de trabalho ${ }^{(18)}$, porque a maioria das lesões podem ser prevenidas com medidas biomecânicas simples tais como o desenho ergonómico dos locais de trabalho, reeducação postural ${ }^{(14)} \mathrm{e}$ o exercício regular ${ }^{(3,8,14)}$ e como a formação nem sempre é eficaz ${ }^{(19)}$, os programas formais de prevenção devem incluir outras estratégias tais como: avaliação da situação de trabalho e intervenção ergonómica; implementação de algoritmos de decisão de apoio à mobilização de doentes; aprendizagem com o erro/incidente; utilização de meios mecânicos de apoio à mobilização e adequação das políticas organizacionais, em particular a nível de recursos humanos ${ }^{(19)}$.

Não podemos deixar de observar que as participantes deste estudo referem que este não é um problema discutido na equipa ${ }^{(12)}$.

As intervenções ergonómicos como a adoção de intervalos regulares de repouso, a colocação dos equipamentos e materiais mais usados por perto para evitar lateralização e torção da coluna, ajustar a cadeira podem facilitar a adoção dos princípios da biomecânica ${ }^{(14)}$, mas não são medidas preventivas suficientes. Qualquer plano de prevenção, tratamento ou reabilitação exige o conhecimento da epidemiologia das doenças músculo-esqueléticas e seus fatores associados ${ }^{(8)}$ e uma intervenção abrangente sobre os fatores de risco ergonómicos, ambientais, individuais e organizacionais.

O resultado de uma revisão sistemática de literatura conclui que a única medida eficaz para diminuir a dor dorsolombar e as lesões é o exercício físico, porém os profissionais não praticam exercício com a regularidade e duração necessária ${ }^{(3)}$. As instituições hospitalares devem ponderar a introdução de tempos para esta prática no horário de trabalho ${ }^{(3,8,14)}$.

Sessões de exercícios curtos e longos de ginástica laboral e yoga durante 10 semanas reduzem as LMELT, melhoram a flexibilidade, a resistência muscular e a condição física ${ }^{(20)}$. Os exercícios de estabilização dos músculos dorsolombares são eficazes no aumento da força e resistência, diminuem a dor/desconforto dorsolombar ${ }^{(3)}$, promovendo o aumento da função e a qualidade de vida dos profissionais.

Os programas de intervenção multifactorial com uma abordagem sistémica e integrada na prevenção das LMELT têm impacto positivo na saúde dos trabalhadores ${ }^{(21)}$. Porém a efetividade das medidas preventivas de LMELT nos EESMO tem sido pouco estudada.

Terminamos conscientes que a necessidade de garantir ações de promoção de saúde funcional, prevenção de alterações estruturais e disfunções/incapacidades, nos profissionais, com ou sem doença de base, e recuperação efetiva das disfunções para a superação das limitações e/ ou restrições impostas por esse tipo de transtorno que afeta a saúde funcional ${ }^{(22)}$, são cruciais para uma verdadeira política de saúde ocupacional e de termos ambientes de trabalho promotores da saúde individual e coletiva.

\section{CONSIDERAÇÕES FINAIS}

Os participantes do estudo, com mais de 9 anos de experiencia profissional, valorizam como medidas preventivas de LMELT o controlo do ambiente físico, a preparação da parturiente, as práticas e comportamentos no período expulsivo e as práticas e comportamentos no período pós-parto.

Verifica-se uma consciência e preocupação com a prevalência das LMELT. Na análise do discurso dos enfermeiros percebe-se o cuidado na avaliação do risco, onde privilegiam os fatores de risco profissional e biomecânicos e em decidir as medidas preventivas em função dessa avaliação, no entanto, não emerge no discurso dos profissionais a apreensão com o controlo dos riscos individuais, físicos e organizacionais.

Apesar de ser possível prever os riscos específicos associados à natureza da atividade profissional e antecipar o 
seu controlo com as práticas preventivas adequadas é difícil manter os princípios da biomecânica ao longo de todo o processo de trabalho de parto.

A análise dos achados permite concluir que os participantes demonstram uma maior preocupação com a segurança e bem-estar dos clientes em detrimento da segurança profissional. $\mathrm{O}$ cuidado com o controlo do ambiente físico, nomeadamente a organização do espaço e manipulação de materiais e equipamentos, são relevadas para segundo plano, apesar da consciência da importância dos riscos físicos e ambientais para a segurança biomecânica.

A mudança rápida e, por vezes, inesperada do comportamento da mulher, bem como, complicações decorrentes do trabalho de parto e diversidade de métodos de trabalho, impele a algumas práticas preventivas das LMELT, como revelam as participantes, na preparação da parturiente ao demonstrarem preocupação na avaliação da capacidade de colaboração da mulher, ao explicitarem os procedimentos a executar antecipando os movimentos e prevendo desta forma o comportamento da mulher.

Nas práticas e comportamentos no período expulsivo, embora esteja presente o recurso aos princípios da biomecânica, a postura dos enfermeiros especialistas são influenciadas pelo tipo de parto e equipamentos, onde sobressai uma permanência de longos períodos de pé e com posturas desconfortáveis, e principalmente pela-segurança da parturiente e bem-estar do recém-nascido.

As participantes revelam na categoria práticas e comportamentos no período pós-parto, uma preocupação com o seu bem-estar individual, ao mencionarem atividades de repouso e mobilidade/deambulação como forma de relaxar os músculos e de desanuviar o stress pós-parto, pois a fadiga e o comportamento individual condicionam práticas seguras.

As dificuldades em manter os princípios da biomecânica são aumentadas pela exiguidade do espaço, materiais e equipamentos danificados.

O controlo deste flagelo de saúde ocupacional, traduzido na elevada prevalência de LMELT, absentismo, incapacidades temporárias e elevados custos, passa por uma cultura organizacional de segurança, que privilegie a formação conjugada com a criação de espaços de trabalho ergonómicamente adaptados e prática de atividade física, ou seja, criando ambientes promotores da saúde individual e coletiva.

Neste sentido, observa-se que a efetividade das medidas preventivas das LMELT, nos EESMOS, não tem sido objeto de estudo bem como, não é uma prática das organizações debaterem esta problemática.

Consideramos que este é um estudo preliminar, de autopercepção, com um número reduzido de participantes o que não permite extrapolar os achados para outras populações.

No entanto, consideramos um contributo importante pois permite-nos identificar lacunas na formação dos profissionais, sobre as medidas preventivas das LMELT e da necessidade de trabalhar as alterações de postura estática para uma postura dinâmica em segurança em sessões de prática simulada de alta fidelidade, quer na formação pósgraduada quer ao longo da vida nos próprios contextos de trabalho, com a necessidade de se elaborarem programas de educação/formação sobre medidas preventivas e estratégias de coping dirigidas a este grupo profissional.

O estudo permitiu ainda identificar áreas de investigação associadas às práticas clínicas e aos comportamentos dos profissionais na utilização dos princípios da biomecânica, bem como a um "olhar" diferente para as instalações e equipamentos das salas de parto.

\section{REFERÊNCIAS}

1. Jellad A, Lajili H, Boudokhane S, Migaou H, Maatallah $\mathrm{S}$, and Frih JBS. Musculoskeletal disorders among Tunisian hospital staff: Prevalence and risk factors. The Egyptian Rheumatologist. 2013;35:59-63.

2. Long $\mathrm{MH}$, Johnston $\mathrm{V}$, and Bogossian F. Workrelated upper quadrant musculoskeletal disorders in midwives, nurses and physicians: a systematic review of risk factors and functional consequences. Applied Ergonomics. 2012;43 (3):455-67.

3. Ellapen TJ, Narsigan S. Work Related Musculoskeletal Disorders among Nurses: Systematic Review. J Ergonomics, 2014,S4:S4-003.

4. Chanchai W, Songkham W, Ketsomporn P, Sappakitchanchai P, and Siriwong W. Prevalence and factors associated with musculoskeletal disorders among Thai hospital orderlies. International Journal of Occupational Hygiene, 2015; 7(3),132-8.

5. Taghinejad H, Azadi A, Suhrabi Z, and Sayedinia M. Musculoskeletal Disorders and Their Related Risk Factors Among Iranian Nurses. Biotech Health Sci. 2016; 3(1):e34473.

6. Chung YC et al. Risk of musculoskeletal disorder among Taiwanese nurses cohort: a nationwide population-based study. BMC Musculoskeletal Disorders 2013;14:144.

7. Filho IMM, Almeida RJ. Estresse ocupacional no trabalho em enfermagem no brasil: uma revisão integrativa. Rev Bras Promoç Saúde, 2016;29(3).

8. Nia HS, Chan YH, Kalantari S, Afshar MH, Taghipour 
B, Kaveh H, and Haghdoost AA. Evaluation of ergonomic factors associated with musculoskeletal disorders in nurses. Adv.Environ.Biol. 2014; 8(6): 3125-9.

9. Long $\mathrm{MH}$, Bogossian FE, and Johnston V. The prevalence of work- related neck, shoulder, and upper back musculoskeletal disorders among midwives, nurses, and physicians: a systematic review. Workplace Health Saf. 2013;61:223-9.

10. Streubert HJ, and Carpenter DR. Investigação qualitativa em enfermagem. ( $5^{\mathrm{a}}$ ed.). Loures: Lusodidacta; 2013.

11. Bardin L. Análise de conteúdo. Lisboa: Edições 70; 2009.

12. Baixinho CL, Presado H, Marques FM, Cardoso M. Prevenção de Lesões Músculo-esqueléticas: relatos dos enfermeiros especialistas em saúde materna e obstetrícia. Atas CIAIQ. 2016;2:488-97.

13. Nevala N, and Ketola R. Birthing Support for Midwives and Mothers- Ergonomic Testing and Product Development. The Ergonomics Open Journal. 2012;5,28-34.

14. Ganer N. Work Related Musculoskeletal Disorders among Healthcare Professional and their Preventive Measure: a report. IJSRSET. 2016;2(4):693-8.

15. Knezevic B, Milosevic M, Golubic R, Belosevic L, Russo A, Mustajbegovic J. Work-related stress and work ability among Croatian university hospital midwives. Midwifery 2011;27:146-53.

16. Razmjou H, Boljanovic D, Lincoln S, Geddes C, Macritchie I, Virdo-Cristello C, and Richards RR. Examining outcome of early physician specialist assessment in injured workers with shoulder complaints. BMC Musculoskeletal Disorders. 2015:16-32.

17. Hodder J, MacKinnon S, Ralhan A, and Keir P. Effects of training and experience on patient transfer biomechanics. Int J Ind Ergon. 2010;40:282-8.

18. Bernal D, Campos-Serna J, Tobias A, Vargas-Prada S, Benavides FG, and Serra C. Work-related psychosocial risk factors and musculoskeletal disorders in hospital nurses and nursing aides: a systematic review and meta-analysis. Int J Nurs Stud. 2015;52(2):635-48.

19. Neves $M$, and Serranheira F. A formação de profissionais de saúde para a prevencção de lesões musculoesqueléticas ligadas ao trabalho a nível da coluna lombar: uma revisão sistemática. Rev Port Saúde Pública. 2014;32(1),89-105.

20. Cheema BS, Houridis A, Busch L, Cheema VR, Melville GW, Marshall PW, Chang D, Machliss B, Lonsdale C, Bowman J, and Colagiuri B. Effect of an office worksite-based yoga program on heart rate variability: outcomes of a randomized controlled trial. BMC Complementary and Alternative Medicine 2013;13:82.

21. Lim H, Black T, Shah S, Sarker S, Metcalfe J. Evaluating repeated patient handling injuries following the implementation of a multi-factor ergonomic intervention program among health care workers. $J$ Safety Res. 2011;42,185-91.

22. Brasil ACO. Promotion of health and human functionality. Rev Bras Promoç Saúde, 2013;26(1):5-8.

\section{Endereço para correspondência:}

Cristina Lavareda Baixinho

Escola Superior de Enfermagem de Lisboa

Avenida Professor Egas Moniz, 1600-190

Lisboa - Portugal

E-mail: crbaixinho@esel.pt 